\title{
THE EFFECT OF PREGNANT MARE'S SERUM GONADOTROPHIN AND HUMAN CHORIONIC GONADOTROPHIN ON THE WET AND DRY OVARIAN WEIGHT OF IMMATURE RATS
}

\author{
E. T. BELL AND S. F. LUNN \\ Medical Research Council, Clinical Endocrinology Research Unit, \\ University of Edinburgh, Edinburgh \\ (Received 18th January 1966)
}

\begin{abstract}
Summary. The effect of the administration of 50 i.u. pregnant mare's serum gonadotrophin (PMSG) alone or the same dosage of this hormone followed $72 \mathrm{hr}$ later by 25 i.u. human chorionic gonadotrophin (HCG) on ovarian wet and dry weight has been investigated in three colonies of rats of the Wistar strain.

Alterations in the ratio of the wet to dry ovarian weight were noted following treatment of PMSG; these were less marked after HCG administration.

The maximum ovarian weight obtained following hormonal treatment varied between the three colonies of rats but the pattern of response was similar. The results did not appear to be influenced by the body weight of the animals.

It is suggested that differences in ovarian weight between the three rat colonies may influence the results obtained in the ovarian ascorbic acid depletion (OAAD) and ovarian cholesterol depletion (OCD) tests.
\end{abstract}

\section{INTRODUGTION}

The effect of the administration of gonadotrophic hormones on the ovaries of immature animals has been studied by various investigators. Thus, the increase in ovarian weight in intact, immature mice has been employed as an assay method for the quantitative determination of PMSG, (Hamburger \& Pedersen-Bjergaard, 1937), while the same end-point in rats and mice treated with large dosages of HCG is thought to be specific for follicle-stimulating hormone (FSH), (Steelman \& Pohley, 1953; Brown, 1955). In recent years, two methods have been described for the assay of luteinizing hormone ( $\mathbf{L H})$, both of which employ intact, immature rats pre-treated with PMSG and HCG. In one of these techniques, the depletion of ovarian ascorbic acid is measured (Parlow, 1958), while in the other the decrease in ovarian cholesterol acts as the end-point (Bell, Mukerji \& Loraine, 1964). The results of all the above assay procedures are calculated on the basis of the wet or total weight of the ovary, while the fluid and tissue content are not generally considered separately. Information 
on the changes in wet weight following the administration of PMSG and HCG to intact, immature rats has been described by Mukerji, Bell \& Loraine (1965); however, comparable results are not available on the effects of these two hormones on ovarian dry weight and the aim of the present communication is to report data obtained from three different animal colonies.

\section{MATERIALS AND METHODS}

Animals

Intact immature female rats of the Wistar strain were obtained from three sources:

Colony A: A closed colony bred in the laboratory in Edinburgh.

Colony B: Rats obtained from Messrs A. J. Tuck, Rayleigh, Essex, England.

Colony $C$ : Animals obtained from the Small Animal Breeding Station, University of Edinburgh.

All the animals varied in age from 21 to 24 days at the beginning of the experiments. Rats from colonies $\mathrm{A}$ and $\mathrm{C}$ ranged in body weight from 30 to $50 \mathrm{~g}$, while those from colony B weighed 40 to $60 \mathrm{~g}$ at this time.

\section{Design of experiment}

A total of fifty-one, fifty and fifty-seven animals obtained from colonies A, $B$ and $C$ respectively received 50 i.u. PMsG (Gestyl: Organon) subcutaneously (s.c.) on Day 0. Groups of rats varying in number from four to thirteen were killed at intervals of $24 \mathrm{hr}$ until the completion of the experiment. Further batches of twenty-four, sixty and ninety-six rats from colonies $A, B$ and $C$ respectively received 50 i.u. PMSG s.c. on Day 0 followed by 25 i.u. HCG (Pregnyl: Organon) s.c. on Day 3. Groups of animals, varying from four to eleven in number, were killed at 24-hr intervals starting on Day 4. Five rats from colony A, which were killed on Day 0, acted as control and received no hormonal treatment. Untreated animals from colonies B and C were killed on Days 0, 3, 9,12 and 15 of the study.

After the animals had been killed by cervical dislocation, the ovaries were removed rapidly by a dorsal approach and dissected free from fat and connective tissue. The wet weight of each ovary was determined to the nearest $0.1 \mathrm{mg}$ in a weighing bottle. The bottles containing the ovaries were then placed in a hot air oven at $110^{\circ} \mathrm{C}$ for a period of 18 to $24 \mathrm{hr}$, at the end of which time they were transferred to a desiccator and allowed to cool to room temperature before being reweighed to the nearest $0.1 \mathrm{mg}$. The weight of the residual tissue was regarded as the dry weight of the ovary and the percentage tissue therefore represents the dry weight expressed in terms of the wet or total ovarian weight. The water content of the ovary is thus the difference between the ovarian wet and dry weight.

\section{RESULTS}

The results of the body weight, ovarian wet weight, ovarian dry weight and percentage tissue following the administration of either PMSG alone or PMSG and HCG for each of the three animal colonies studied are shown in Text-figs. 1 to 3. 
During the period of the experiment the body weight of the control and treated animals in colonies B and $\mathbf{C}$ was similar. A slight rise in both ovarian wet and dry weight occurred in the untreated animals in these two colonies but the values obtained were considerably lower than those in the treated groups at all stages of the study.

Colony $A$ (Text-fig. 1). Following the administration of PMSG a slight but significant fall in body weight occurred $(P<0.01)$. Subsequently the weight of

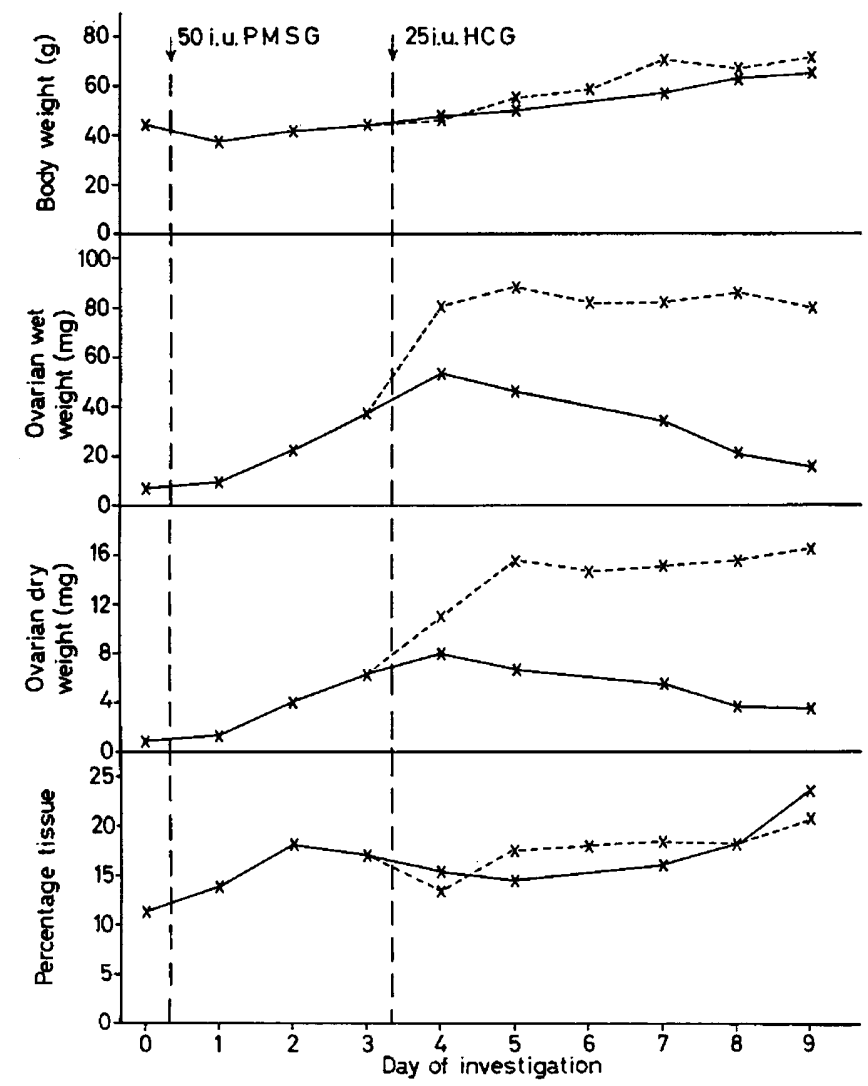

TEXT-FIG. 1. The effect of PMSG alone (-), and of PMSG and HCG (- - -) on rat ovaries; colony A.

the animals increased steadily and no marked effect was noted after the injection of HGG except on Day 7 when the weight of the group treated with both PMSG and HGG was significantly higher $(0.02>P>0.01)$ than that given PMSG alone.

Ovarian wet weight increased after PMSG administration reaching a maximum of $53.2 \mathrm{mg}$ on Day 4 ; thereafter values fell during the remainder of the experiment. Within $24 \mathrm{hr}$ of the injection of HGG a significant increase $(P<0.001)$ in ovarian wet weight occurred and high figures were noted from Days 5 to 9 . The pattern of ovarian dry weight was similar to that of ovarian wet weight.

Within $48 \mathrm{hr}$ of the injection of PMSG a significant rise $(0.05>P>0.01)$ in the percentage tissue had occurred representing a relative increase in the dry as 
compared with the wet weight of the ovary. This was followed by a slow fall for 3 days and by a subsequent rise. Following the administration of HCG the percentage tissue showed a tendency to increase.

Colony $B$ (Text-fig. 2). The body weights of the control and treated groups fluctuated somewhat but in general were similar. Following the administration

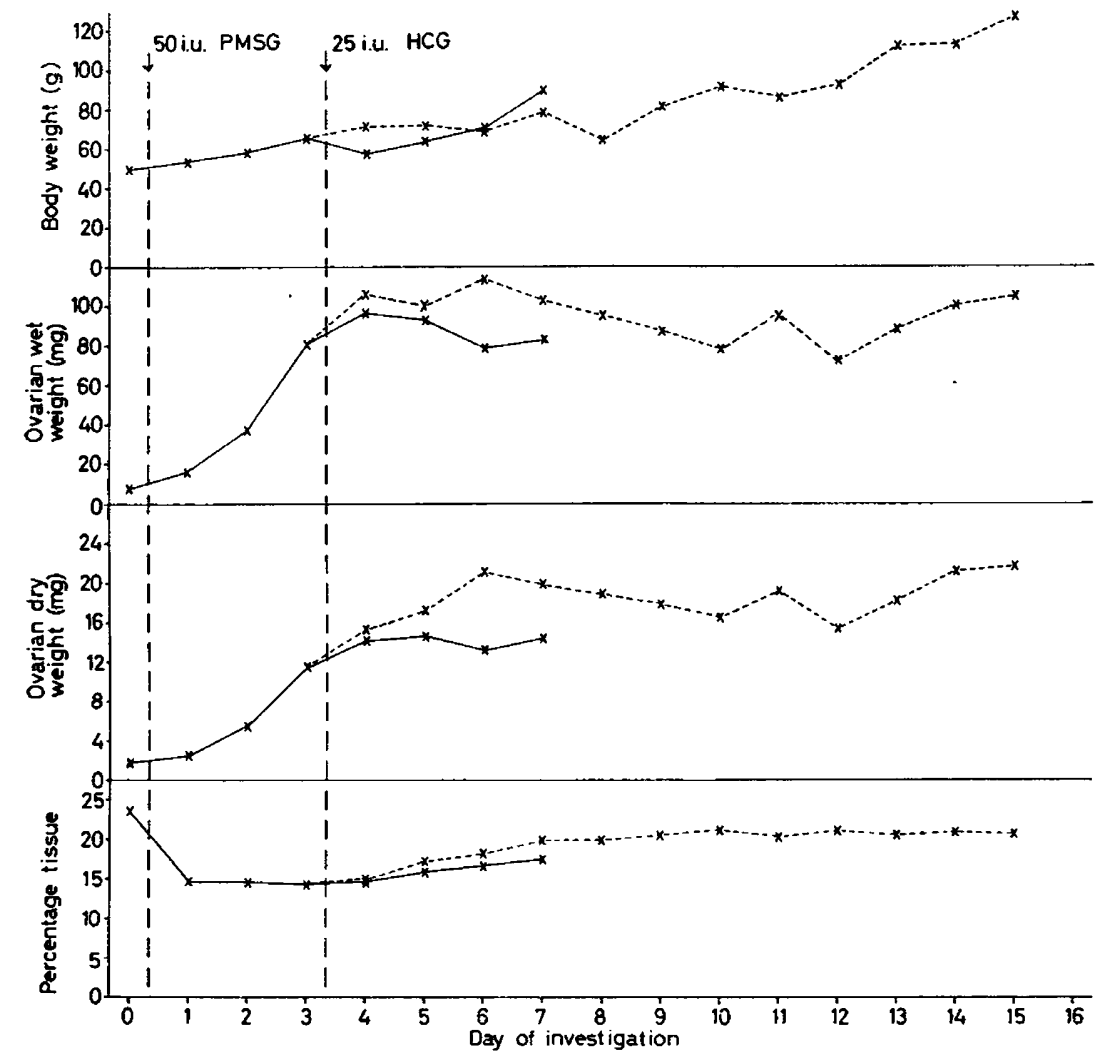

TEXT-FIG. 2. The effect of PMSG alone (-), and of PMSG and HCG (- - -) on rat ovaries; colony $\mathbf{B}$.

of PMSG the pattern of ovarian wet weight was similar to that in colony A: a maximum level of $97.0 \mathrm{mg}$ being noted on Day 4. When HCG was given in addition to PMSG the ovarian weight remained high although some variations occurred. It should be noted that the pattern of ovarian dry weight resembled that of ovarian wet weight.

Within $24 \mathrm{hr}$ of the administration of PMSG a marked fall $(P<0.001)$ in the percentage of ovarian tissue had occurred. Subsequently levels remained constant for 3 days and then rose gradually during the remaining 3 days of the study. When HCG was given, the percentage tissue increased until Day 7 and thereafter remained relatively constant, being similar to the values obtained for the untreated animals.

Colony $C$ (Text-fig. 3) After the injection of PMSG the significant fall in body weight which occurred on Day $1(0.01>P>0.001)$ was followed by a steady rise 
during the remainder of the study. The pattern of ovarian wet weight after the administration of PMSG was similar to that in colonies A and B. The highest ovarian weight $(71.4 \mathrm{mg})$ was noted on Day 4 . The injection of HCG caused a further increase and subsequent maintenance of ovarian wet weight.

After injection of PMSG the ovarian dry weight increased for 5 days reaching a maximum of $10.4 \mathrm{mg}$ and then decreased. The injection of HCG caused a

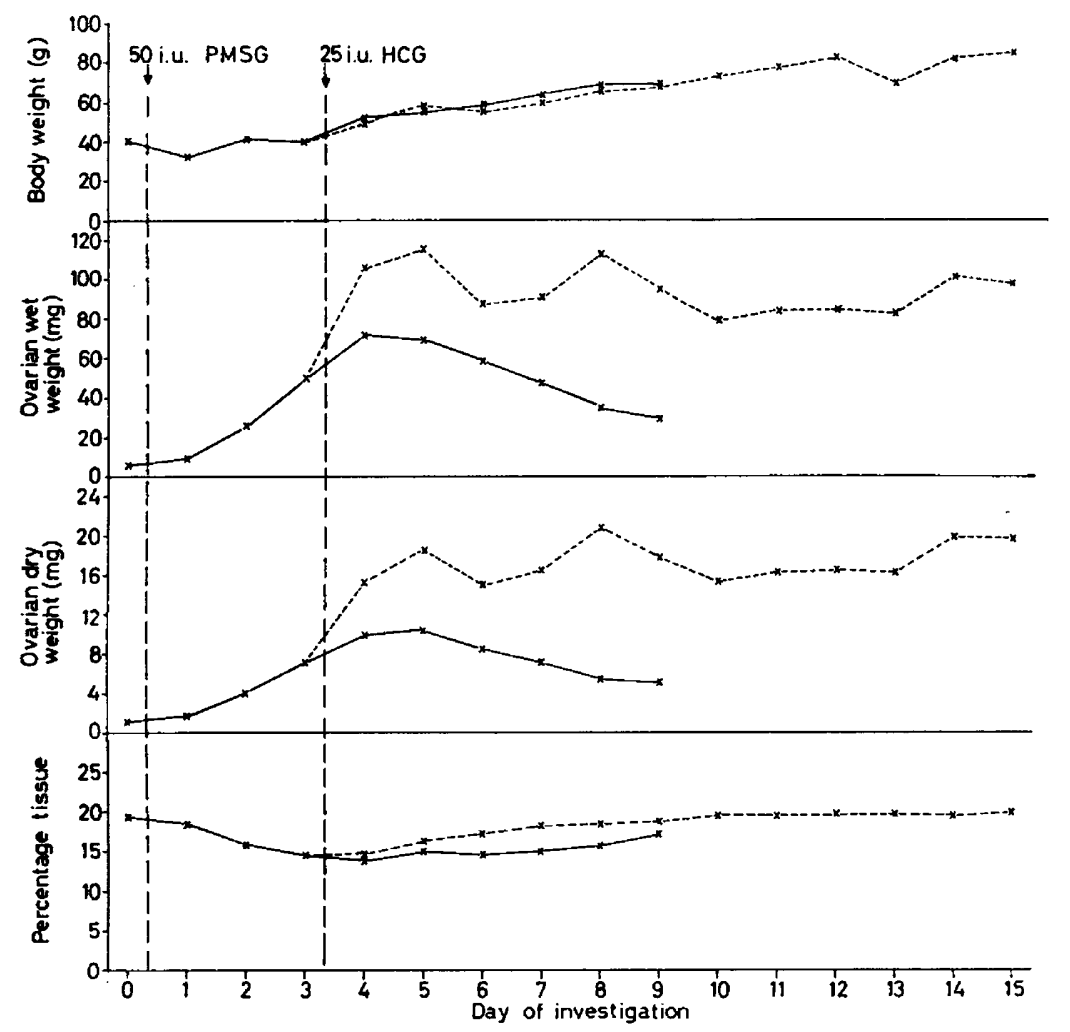

TEXT-FIG. 3. The effect of PMSG alone (-), and of PMSG and HCG (- . - -) on rat ovaries; colony G.

significant increase in ovarian dry weight $(0.01>P>0.001)$ and the high levels were subsequently maintained.

A slow fall in the percentage ovarian tissue occurred from Days 2 to 4 after the administration of PMSG; this was followed by relatively constant values. In animals which received both PMSG and HCG a steady rise in the percentage tissue occurred from Day 4 to Day 10, thereafter no alteration was noted. In general, the values observed in the untreated animals were higher than those in the PMSG group, but were similar to those in the animals receiving PMSG and HCG.

\section{DISGUSSION}

At the commencement of the study the body weight of the rats in colonies $\mathrm{A}$ and $\mathrm{C}$ was approximately $10 \mathrm{~g}$ lower than that of $\mathrm{B}$. A transient but significant 
fall in body weight occurred in colonies $\mathrm{A}$ and $\mathrm{C}$ following the administration of PMSG; however, apart from this, hormonal treatment appeared to have no marked effect. It should be noted that the weight gain of all three colonies, when expressed as a percentage, was similar during the period of study; accordingly, it is suggested that the body weight of the animals did not materially affect the ovarian weight obtained following hormonal administration.

The present investigation has confirmed that the administration of a single injection of 50 i.u. PMSG to intact immature rats stimulates ovarian growth (see Mukerji et al., 1965). It has also been shown that the period of weight increase lasts for approximately 4 days and that subsequently the ovaries regress. However, the effect of the hormone on the wet weight was not the same as that on the dry weight of the ovary in all three colonies. In colony $A$ the percentage tissue rose initially indicating a relative reduction in the fluid content; this was followed by a slow fall and by a second increase. In both colonies $\mathrm{B}$ and $\mathrm{C}$ there was an initial fall and subsequent rise in the percentage tissue.

The administration of 25 i.u. HCG to rats treated with 50 i.u. PMSG $72 \mathrm{hr}$ previously resulted in ovulation, corpus luteum development and luteinization of unruptured follicles (Bell \& Lunn, unpublished observations). The injection of HCG was also associated with a further increase and subsequent maintenance of ovarian weight as compared with the rats treated with PMsG alone. The pattern of ovarian wet and dry weight was similar in the three colonies studied. This is illustrated by the slow rise in percentage tissue which occurred from Days 4 to 9 of the study and by the absence of any change after this time.

In each colony, the wet weight of the untreated ovaries was similar at the beginning of the experiment, but the dry weight was lower in colony A than in B or C. During the remainder of the study the ovarian weight of the untreated animals in colony B increased more rapidly than did that in colony C. Despite the similarities in the pattern of response to hormonal treatment in the three groups of animals, the absolute ovarian weights obtained were, however, different. Following PMSG administration, the highest ovarian wet and dry weight obtained in colony A was lower than that in the other two colonies. Similar results were noted in the animals which received both PMSG and HCG. The explanation of these findings is obscure, but in view of the fact that the experiments reported herein were carried out under similar environmental conditions it seems unlikely that such conditions could account for the variations observed. A further possibility is that differences in the blood level of endogenous gonadotrophin in the three colonies could influence the results obtained.

The conditions selected for treatment with PMSG and HCG were identical with those employed for the OAAD and OCD assays in this laboratory (SchmidtElmendroff \& Loraine, 1962; Bell et al., 1964; Bell, Loraine, Mukerji \& Visutakal, 1965). According to the specifications of the originators, the bioassay proper should be conducted on the 5 th to 9 th and on the 11 th days after HCG administration in the OAAD and OCD tests respectively. The fact that the ovarian weight at the time of assay varied considerably between the colonies suggests that the pre-treatment conditions used may not necessarily have been 
optimal. It seems possible that experiments undertaken to assess the dosage of PMSG and HCG required will be necessary in the establishment of the OAAD and $O C D$ tests in any given laboratory. In the present experiment there was no evidence of marked variation in the percentage ovarian tissue between the 5 th and 11 th days after HCG. This finding suggests that no additional information will be obtained by expressing the results of assays by the OAAD and OCD methods in terms of the dry, rather than the wet, weight of the ovary. However, the possibility remains that it might be of value to calculate the data of such assays in terms of lipid-free dry weight or protein nitrogen, and this problem is at present under investigation.

\section{ACKNOWLEDGMENT}

The authors wish to thank Organon Laboratories Ltd for supplies of PMSG and HCG.

\section{REFERENCES}

Bell, E. T., Loraine, J. A., Mukerjl, S. \& Visutakul, P. (1965) Further observations on the ovarian ascorbic acid depletion test for luteinizing hormone. F. Endocr. 32, 1.

Bell, E. T., MukerJi, S. \& Loraine, J. A. (1964) A new bioassay method for luteinizing hormone depending on the depletion of rat ovarian cholesterol. F. Endocr. 28, 321.

BRown, P. S. (1965) The assay of gonadotrophin from urine of non-pregnant human subjects. 7 . Endocr. 13, 59.

Hamburger, C. \& Pedersen-Bjergaard, K. (1937) The assay of gonadotrophic hormones. Standardization curves for pregnant mare's serum hormone and human pregnancy hormone. $Q . \mathcal{F}$. Pharm. Pharmac. 10, 662.

MukerJi, S., BeLl, E. T. \& Loraine, J. A. (1965) The effect of pregnant mare serum gonadotrophin and human chorionic gonadotrophin on rat ovarian ascorbic acid and cholesterol. F. Endocr. 31, 197.

PArlow, A. F. (1958) A rapid bioassay method for LH and factors stimulating LH secretion. Fedn Proc. Fedn Am. Socs exp. Biol. 17, 402.

Schmidt-Elmendorf, H. \& LoRAine, J. A. (1962) Some observations on the ovarian ascorbic acid depletion method as a test for luteinizing hormone activity. F. Endocr. 23, 413.

Steelman, S. L. \& Pohley, F. M. (1953) Assay of the follicle-stimulating hormone based on the augmentation with human chorionic gonadotrophin. Endocrinology, 53, 604 . 